\title{
Spinal Schwannoma as \\ Pregnancy Related Spinal Tumor
}

Kadek Dede Frisky Wiyanjana', Kevin Kristian Putra',

\section{Sri Maliawan²,}

I Made Gotra ${ }^{3}$

'Medical Education Program,

Faculty of Medicine, Udayana University,

Bali, Indonesia

${ }^{2}$ Department of Neurosurgery,

Faculty of Medicine Udayana University,

Sanglah General Hospital, Denpasar, Indonesia

${ }^{3}$ Department of Pathological Anatomy,

Faculty of Medicine Udayana University,

Sanglah General Hospital, Denpasar, Indonesia

\begin{abstract}
Spinal schwannoma is the most common benign spinal tumors that originated from peripheral nerve sheath tumors. Male and females are equally affected. Due to hormonal imbalance during pregnancy, it might affect the release of the tumor-growth factor. The symptoms usually start with pain and sensory loss at the spinal cord level. We report a case of thoracolumbar schwannoma with a history of chronic low back pain since post-pregnancy. A Thirty-Seven years old female suffered chronic low back pain for three years since she had a pregnancy. In the last four months, she suffered paresis and tingling sensation on the right foot. MRI has shown intradural mass at Th 12 - L2. Biopsy, tumor resection, and laminectomy were performed to do the histopathology analysis, resect, and stabilization. Histopathology examination revealed Schwannoma types. The patient was discharged three days after complete tumor resection and stabilization, with an improvement of neurological function. Spinal Schwannoma can occur as a Pregnancy-related spinal tumor (PRST); it can be diagnosed during pregnancy or within a year after delivery. The incidence is rare. Therefore proper workup and initial examination are needed and followed by careful and considerate treatment.
\end{abstract}

Keywords: Spinal Schwannoma, Pregnancy-related spinal tumor, Spinal tumor.

\section{Introduction}

Spinal Schwannoma is the most common benign tumors that originated from peripheral nerve sheath tumors, which also include neurofibromas, perineuriomas, granular cell tumors, and malignant nerve sheath tumors. ${ }^{1}$ The natural history of schwannomas are slow-growing, solitary, usually attached to peripheral nerves, and consisting of a clonal population of Schwann cells, which often undergo cystic and degenerative changes. ${ }^{2}$ Due to its characteristics, schwannomas or neurilemmoma, represent approximately $30 \%$ of all primary spinal cord tumors. The incidence of spinal schwannoma was $0.3-0.4 / 100,000$ cases annually, either in solitary or syndromic form. Males and females appeared to be equally affected and the onset can be in productive age or late, approximately 25-60 years old. ${ }^{1-4}$

The occurrence of schwannoma in women, especially in pregnancy, are reported. Due to hormonal changes in pregnancy may influence the slow-growing schwannoma growth. ${ }^{5-6} \mathrm{Al}-$ though the incidence is rare. A pregnancy-related spinal tumor (PRST) occurs in about 1 in 1000 to 2000 pregnancies and usually diagnosed during pregnancy or within a year after delivery. Giant cell tumor, hemangioma, and schwannoma are common types of PRST. The symptoms of PRST were usually vague. ${ }^{7-8}$ It may take months to years to finally symptomatic. The neurological deficit was progressive. It starts from pain, a sensory loss like paresthesia and the least
Corresponding author: Kadek Dede Frisky Wiyanjana Medical Education Program, Faculty of Medicine, Udayana University, Bali, Indonesia dedewiyanjana@gmail.com 
common were motor deficit of sphincter function. ${ }^{1-4}$

We reported a case of post-delivery patient with 3 years chronic low back pain after delivery, with progressive neurological deficit in the last 3 months and diagnose with schwannoma at thoracolumbar vertebrae.

\section{Case Report}

A 37-year-old female came to the hospital with gradual weakness on both limbs for the last four months. Initially, a patient only felt weakness on the right limb and had difficulty when walking, but since the last two months, it was getting worse until the patient unable to walk and feel completely weakness on both limbs completely. Patient sometimes felt tingling sensation down to the right limb along with the weakness of the right limb in the last four months. The patient had a history of chronic low back pain for the last three years since she had pregnancy and labor in late 2015. Pain mostly felt at the back, there was no spreading pain to the limbs. Back pain was not associated with an increase or decrease activity, it happens spontaneously. For this pain patient only check up to the general practitioner doctor \& physiotherapy and only treatment for the pain reliever.

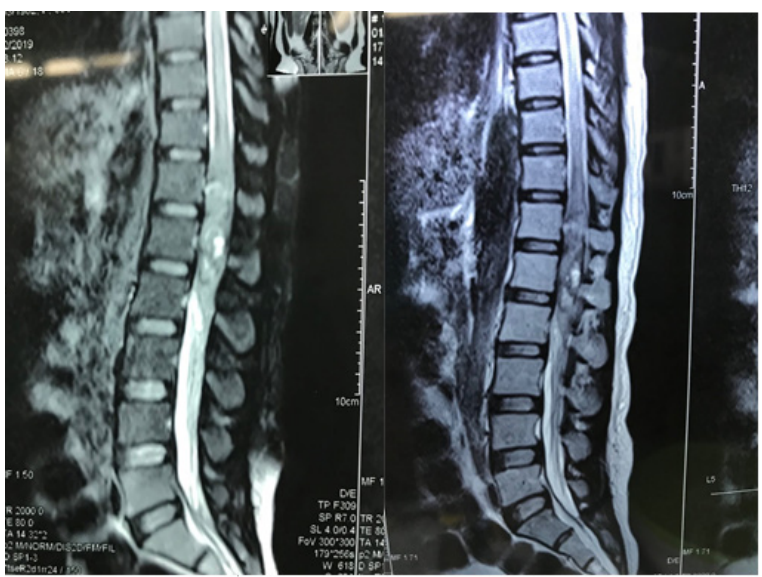

Fig 1. Left: Sagittal T2-weighted MRI image of a 23-year-old patient showing mixed-intensity lesion at the Th12-L2 level, in front of beginning conus medullaris. Right: Sagittal T1-weighted MRI.

No history of problems in defecation and urination, no traumatic accident, no other previous disorders such as fever, weight loss, neurofibromatosis or specific skin lesions.

From physical examination, vital sign and general examinations were normal. Neurological examination found that motoric strength of both lower limbs was decreased into 2 (MMT Scale). Sensory examination founding was Segmental Paresthesia below Th-12 dermatome. Sagittal T2-weighted MR image, shown in Fig 1, revealing a mixed-intensity mass, hypointense \& isointense image, lesion filling the spinal canal at Th 12- L2 segmental level. It demonstrates a mass with solid and cystic components. The patient underwent a tumor resection, biopsy, and spine stabilization with posterior approach. After three days of surgery, patient was discharge with improve neurological examination. The histology examination (shown in Fig 2) confirms the spinal tumor was schwannoma with evidence of Atoni A, Atoni B in the mass and Verocay Bodies.

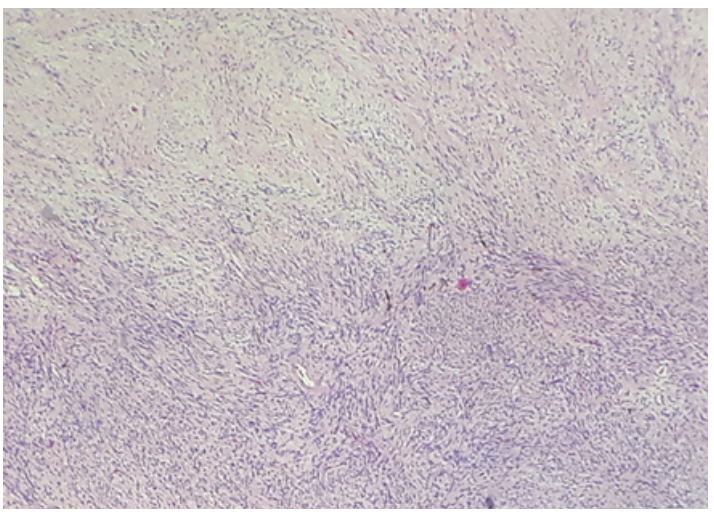

Fig 2. Photomicrograph showing fibrous tissue which comes from the proliferation of neoplastic cells that constructs the hypercellular region (Atoni A) and Hipocelluler region (Atoni B). Cells in the Hipercelular area had spindle wavy core, bland chromatin, regular nucleus, sitosplasma eisonifilik. Nuclear palisading around the fibrillary process (Verocay bodies) is seen.

\section{Discussion}

Solitary intradural-extramedullary spinal schwannoma in the thoracolumbar with history of chronic low back pain after giving birth was rare. the symptoms were had similar characteristics as reported in some literature and reports. They are slow-growing benign tumors, typically encapsulated, and composed entirely of well-differentiated Schwann cells. In most cases, schwannomas are solitary and sporadic, sometimes it can be multiple and often associated with neurofibromatosis type 2 (NF2) and schwannomatosis. ${ }^{2}$ The location of spinal schwannoma were not specific in spinal region. The site were $38 \%$ localized to the cervical spine, $28 \%$ to the thoracic spine, and $34 \%$ to the lumbosacral spine. ${ }^{9-10}$ The characteristics of lumbar nerve roots, which run long distances from the conus to the foramina, might be the reason why lumbar is more common, as spinal Schwannoma arise in most cases from the dorsal rootlets as they develop from Schwann cells. $^{2}$

The Pain was the first symptoms that occur in spinal schwannomas and another spinal related tumor. Then followed by paresthesia, numbness, and motoric weakness. Less common symptom was impairment of sphincter function. In this patient, the low back pain with uncertain causes and was not relieved after series of pain killer. This was one of the red flags for low back pain. We should think possibility of spinal tumor, to get early screening and detection, beside other condition like history of post-pregnancy.

A pregnancy-related spinal tumor (PRST) occurs in about 1 in 1000 to 2000 pregnancies and usually diagnosed during pregnancy or within a year after delivery. In this case, we found an intradural extramedullary thoracolumbar schwannoma in women, years after delivery, with chronic low back pain and motoric deterioration as the initial symptoms. The common PRST were giant cell tumors, hemangiomas, ependymoma, schwannomas, and metastases. ${ }^{11}$ There were different theories as to why pregnancy might trigger the growth of the spinal tumor. Some reported that estrogen and progesterone levels, 
which elevated during pregnancy, can accelerate the growth of the tumor. The increased level of growth factors and angiogenic factors could accelerate the tumor growth for giant cells tumors, ependymoma, schwannoma, and metastases. Other than the biochemical changes in pregnancy, hemodynamic can also play the part, especially in hemangioma the venous pooling, that occur by the increased blood flow volume through the vertebral venous plexus secondary to compression of the vena cava by the uterus can cause the increase of the spinal compression. ${ }^{3,7-11}$

On Histopathological Examination, the tumor was a well-encapsulated mass with white-greyish color. There was no gross hemorrhage within. Microscopically, tumor was well-defined encapsulated with fibrous tissue which comes from the proliferation of neoplastic cells that constructs the hypercellular region (Atoni A) and Hypocellular region (Atoni B). Cells in the hypercellular area had spindle wavy core, bland chromatin, regular nucleus, and eosinophilic cytoplasm. Nuclear palisading around the fibrillary process (Verocay bodies) was seen. Morphology diagnosis is Schwannoma.

\section{Conclusion}

Neurological deficit after delivery that caused by spinal tumor might happen due to physiological changes in before and after delivery to rumor growth. Schwannoma spine tumor, as the most common intradural tumor, could occur as a Pregnancy-related spinal tumor (PRST) and it can be diagnosed during pregnancy or years after delivery. The incidence is rare, therefore proper and considerate workup and early examination are needed, followed by careful and considerate treatment.

\section{Acknowledgement}

None

\section{References}

1. Junior S, Silva R, Brito J, et al. Diagnosis and therapeutic management updated on intrathoracic schwannoma. Archives of Clinical and Experimental Surgery (ACES). 2017;6(4):1. DOI : 10.5455/ aces. 20170104051917.

2. Samii M, Giordano M. Spinal Schwannomas. Spinal Cord Tumors. 2019;161-169. DOI : 10.1007/978-3-319-99438-3_10.

3. Hilton D, Hanemann C. Schwannomas and Their Pathogenesis. Brain Pathology. 2014;24(3):205-220. DOI : 10.1111/bpa.12125.

4. Tacken M, Ramakers B, Idema A, et al. Unexpected schwannoma un- masked by the physiologic changes of pregnancy. Clinical Case Reports and Reviews. 2016;2(8). DOI: 10.15761/CCRR.1000261.

5. Başaran Gündoğdu E, Ekici M. Intradural extramedullary cystic schwannoma of the cervical spine. The European Research Journal. 2019;5(3):572-575. DOI: 10.18621/eurj.384041.

6. Nayak R, Chaudhuri A, Ghosh S, et al. Thoracic intramedullary schwannoma: A case report and review of literature. Asian Journal of Neurosurgery. 2015;10(2):126. DOI : 10.4103/1793-5482.145155.

7. Meng T, Yin H, Li Z, et al. Therapeutic Strategy and Outcome of Spine Tumors in Pregnancy. Spine. 2015;40(3):146-153. DOI: 10.1097/ BRS.0000000000000719.

8. Chen R, Xiao A, Xing L, et al. A rare thoracic intraspinal schwannoma in twin pregnancy with aggravated clinical presence. Medicine. 2017;96(11):e6327. DOI : 10.1097/MD.0000000000006327.

9. Safaee, M, Parsa A, Barbaro N, et al. Association of tumor location, extent of resection, and neurofibromatosis status with clinical outcomes for 221 spinal nerve sheath tumors. Neurosurgical Focus. 2015;39(2):E5. DOI : $10.3171 / 2015.5$.FOCUS15183.

10. Peng Li, Fu Zhao, Jing Zhang, et al. Clinical features of spinal schwannomas in 65 patients with schwannomatosis compared with 831 with solitary schwannomas and 102 with neurofibromatosis Type 2: a retrospective study at a single institution. J Neurosurg Spine. 2015;24(1):110. DOI: 10.3171/2015.3.SPINE141145.

11. Van der Hoeven M, de Pont L, Koppen H. Severe Nightly Thoracic Pain Presenting during Pregnancy: A Case Report. Case Reports in Neurology. 2018;10(2):135-139. DOI : 10.1159/000488756. 